\title{
The Status of the ACRF Millimeter Wave Cloud Radars (MMCRs), the Path Forward for Future MMCR Upgrades, the Concept of 3D Volume Imaging Radar and the UAV Radar
}

\author{
P. Kollias, M. Miller \\ Brookhaven National Laboratory \\ K. Widener, R. Marchand, T. Ackerman \\ Pacific Northwest National Laboratory
}

December 2005

Work supported by the U.S. Department of Energy,

Office of Science, Office of Biological and Environmental Research 


\section{DISCLAIMER}

This report was prepared as an account of work sponsored by the U.S. Government. Neither the United States nor any agency thereof, nor any of their employees, makes any warranty, express or implied, or assumes any legal liability or responsibility for the accuracy, completeness, or usefulness of any information, apparatus, product, or process disclosed, or represents that its use would not infringe privately owned rights. Reference herein to any specific commercial product, process, or service by trade name, trademark, manufacturer, or otherwise, does not necessarily constitute or imply its endorsement, recommendation, or favoring by the U.S. Government or any agency thereof. The views and opinions of authors expressed herein do not necessarily state or reflect those of the U.S. Government or any agency thereof. 


\section{Executive Summary}

The United States (U.S.) Department of Energy (DOE) Atmospheric Radiation Measurement (ARM) Climate Research Facility (ACRF) operates millimeter wavelength cloud radars (MMCRs) in several climatological regimes. The MMCRs, are the primary observing tool for quantifying the properties of nearly all radiatively important clouds over the ACRF sites. The first MMCR was installed at the ACRF Southern Great Plains (SGP) site nine years ago and its original design can be traced to the early 90s. Since then, several MMCRs have been deployed at the ACRF sites, while no significant hardware upgrades have been performed. Recently, a two-stage upgrade (first C-40 Digital Signal Processors [DSP]-based, and later the PC-Integrated Radar AcQuisition System [PIRAQ-III] digital receiver) of the MMCR signal-processing units was completed.

Our future MMCR related goals are: 1) to have a cloud radar system that continues to have high reliability and uptime and 2) to suggest potential improvements that will address increased sensitivity needs, superior sampling and low cost maintenance of the MMCRs. The Traveling Wave Tube (TWT) technology, the frequency (35-GHz), the radio frequency (RF) layout, antenna, the calibration and radar control procedure and the environmental enclosure of the MMCR remain assets for our ability to detect the profile of hydrometeors at all heights in the troposphere at the ACRF sites.

In the near-term (0-2 years), the ACRF should proceed with the upgrade of the remaining MMCR with the PIRAQ-III digital receiver. The upgrade (completed in early 2007) will bring commonality among the 5 operational MMCR at the ACRF sites, reduce the maintenance costs, and provide uniform temporal resolution and radar operational modes in all the ACRF sites. Each signal processing unit upgrade is estimated to $\sim \$ 30 \mathrm{~K}$ per MMCR.

In the long term (2-5 years) the ACRF should pursue the development of a high-performance digital data processor and timing controller implemented in field-programmable gate arrays (FPGA) that will replace the existing intermediate frequency (IF) hardware/software and timing MMCR components. The digital radar transceiver will replace the analog radar waveform generator that generates the pulse compression (frequency modulation of the transmitted waveform technique that results to higher sensitivity) with a direct digital synthesis (DDS) PC-based card. Furthermore, the current IF section of the MMCR will be replaced with an FPGA-based digital receiver that will offer higher flexibility, direct control of the radar waveform through the DDS, and higher overall MMCR performance.

This development can be the focus of DOE's Small Business Innovation Research (SBIR) (proposed in 2006, completed in 2008), with an objective to develop a digital radar transceiver and timing unit suitable for ground-based cloud and precipitation profiling radars that utilizes chirp pulse waveforms and fast Fourier transform (FFT) processing for superior performance and multi-functionality. This development should be the outcome of strong interaction between the ARM atmospheric radiation and cloud physics scientists and small business specialists, and will lead to a more robust, flexible and reliable future MMCR design and also help the development of "in-house" expertise on advance digital radar transceiver technology.

However, the development of such a digital transceiver raises important development challenges. Currently, the ACRF has no hot-spare MMCR that can be used for the development and testing of the 
next generation digital transceiver. Furthermore, the limited technical capabilities at the ACRF sites prevent strong intervention in the MMCR hardware/software by the locals. Thus, it is important that we have a continuous and consistent monitoring of the ACRF MMCR health and operational status. The MMCR mentor should schedule annual site visit for calibration inspection, enhance work with the MMCR sites, and develop algorithms for ascertaining trends related to the operational status of the MMCR. Currently, the mentor is under funded to do the aforementioned tasks. ARM scientists must maintain contact with this new, low cost, and powerful radar technology, since it can lead to the development of new sophisticated and innovative radar tools that can address particular scientific objectives such as the 3-D mapping of the cloud field or high-resolution cloud imaging.

Furthermore, in the next 2-3 years, we should consider refurbishing the MMCR antennas. The MMCR antenna refurbishing is a time-consuming effort that involves extensive antenna transportation time from the remote sites to the refurbishing location. We need to purchase a spare antenna (6-foot diameter, $\$ 50 \mathrm{~K})$ that will rotate through the ACRF site and replace the antenna that will be sent for refurbishing. This will allow us to minimize the radar down-time during this process. The estimated refurbishing cost per antenna is $\sim \$ 30 \mathrm{~K}$ ( and more for SGP). The MMCR antenna refurbishing project could start after we have completed the PIRAQ-III upgrades at the SGP and North Slope of Alaska (NSA) sites and thus spread the financial burden over several years.

The acquisition of new radars by the ACRF to enhance the information extracted by the existing radars at the ACRF sites is proposed. Emphasis is given to a new rapid-scan radar system with the ability to characterize the 3-D structure of clouds and precipitation. This multi-functioning three-dimensional (3D) Volume Imaging Radar (3D-VIR) system will address the need for mapping the 3D cloud structure over an extensive area (40x40x20 km volume box - "local scale") around the ACRF SGP central facility. The system concept of the 3D-VIR explores the use of new emerging radar technologies such as the use of phased array antennas and the use of pulse compression that can add agility and multi-purpose design to the 3D-VIR. The 3D-VIR phased-array 9.4-GHz radar system will be a multi-tasking and multifunctioning radar that: 1) produce 3-D cloud imaging in a 40x40x20 $\mathrm{km}$ box around the ACRF SGP site and 2) provide adaptive/selective scanning of cloud elements during intensive observation periods (IOPs). The cost of the 3D-VIR is around $\$ 1.5 \mathrm{M}$.

The development of suitable cloud radar remains a current need for the ARM-UAV Program. Although the term UAV refers to an unmanned airborne vehicle, the Proteus is piloted aircraft and its operational characteristics, space, weight and power consumption requirements are different from that of future UAV. For airborne cloud radar, the physical space, weight and power limitations are critical to the radar type selection and design (e.g., frequency, antenna, transmitter type). Thus it is important that the characteristics of the ARM-UAV are updated to the current and future trends of UAVs. The initial effort for the acquisition of a UAV cloud radar through the collaboration between the UMass-Microwave Remote Sensing Laboratory (MIRSL) and DOE was not successful because of a lack of sufficient knowledge for the development and design of high-altitude, compact radar systems. Ten years later, the plethora of airborne cloud radar developments from other agencies (e.g., the National Science Foundation [NSF], National Aeronautics and Space Administration [NASA] and National Research Council [NRC] Canada) offers significant advancements (e.g., pulse compression, EIK transmitters, digital receivers) in the radar design concepts of airborne cloud radars. These ideas should be incorporated in the design phase of the ARM-UAV cloud radar. 


\section{Contents}

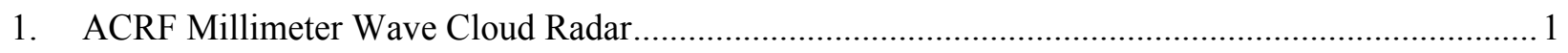

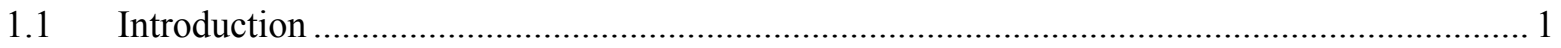

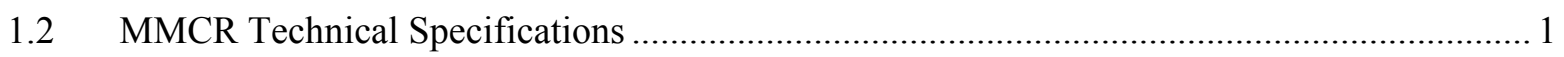





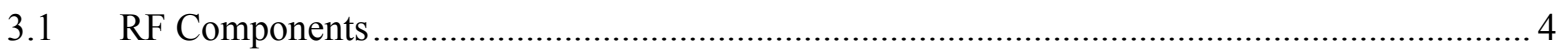

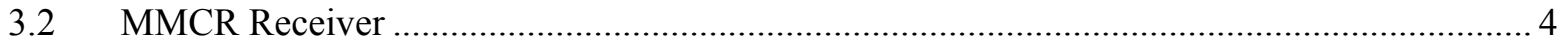

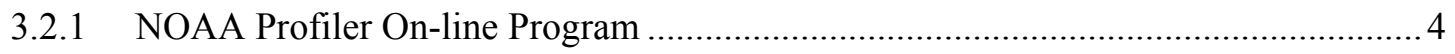



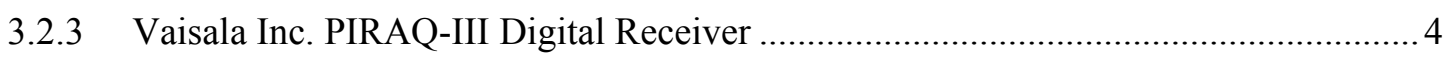

3.2.4 Present Status of MMCR Receivers .................................................................... 5

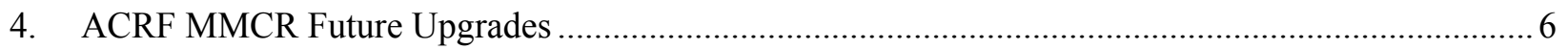

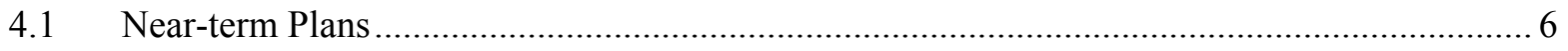

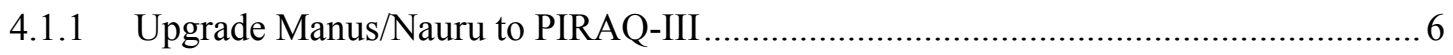

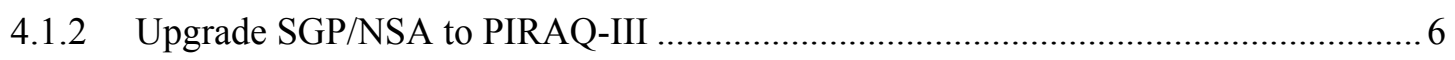

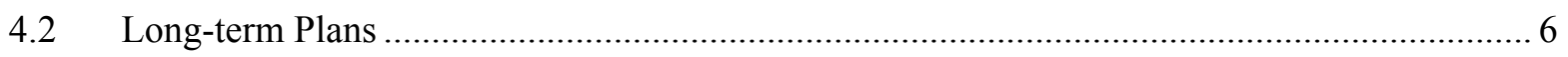

4.2.1 Digitally Generated Pulse Compression............................................................... 6

5. Path Forward for the Acquisition of New ACRF Radars.............................................................. 9

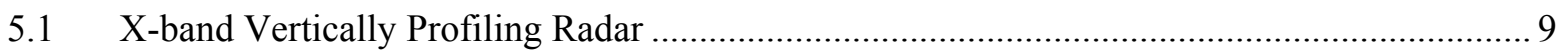

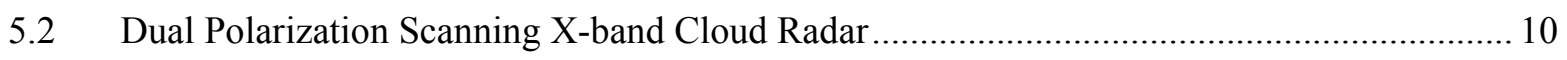

5.3 3-D Volume Imaging Radar - System Concept .............................................................. 11

6. ARM - Unmanned Aerospace Vehicle Cloud Radar .................................................................. 14

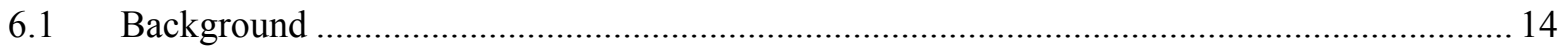

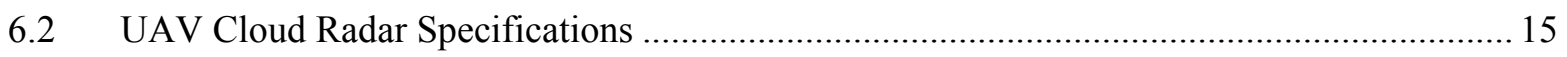



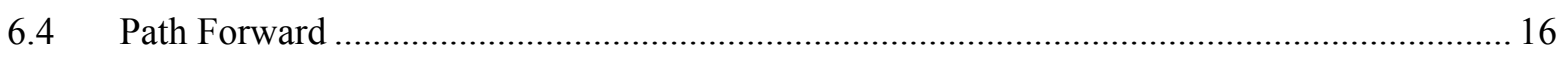

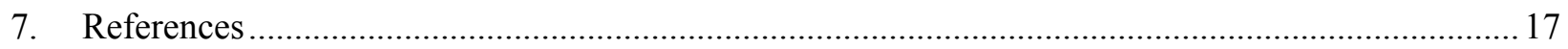




\section{Figures}

1. Past, present and future upgrades of the SCRF MMCR receiver ................................................ 5

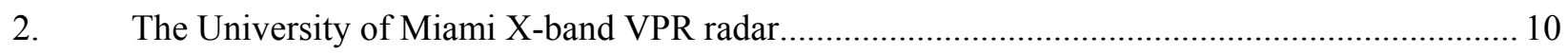

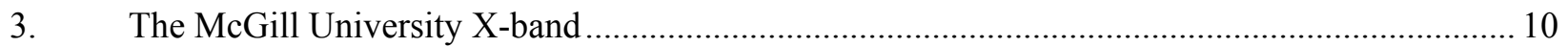

4. The NOAA/D X-band scanning and polarimetric radar ........................................................... 11

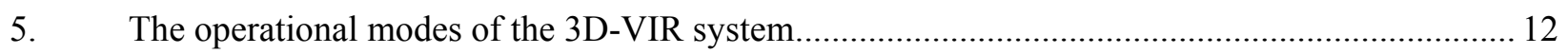

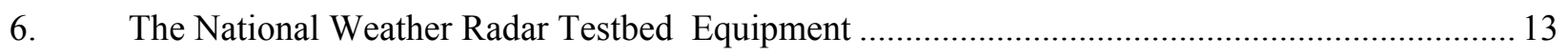

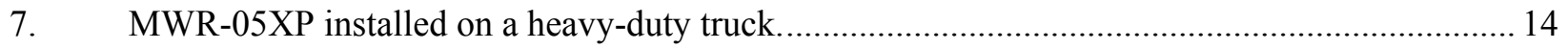




\section{ACRF Millimeter Wave Cloud Radar (MMCR)}

\subsection{Introduction}

The United States (U.S.) Department of Energy (DOE) Atmospheric Radiation Measurement (ARM) Climate Research Facility (ACRF) operates millimeter wavelength cloud radars (MMCRs) in several climatological regimes. These vertically-pointing cloud profiling radars supply the three most important Doppler spectrum moment estimates, which are the radar reflectivity, or zero moment, the mean Doppler velocity, or first moment, and the Doppler spectrum width, or second moment, as a function of time and height. The ACRF MMCR (Moran et al., 1998) Doppler moment estimates are the most important source of information we have for the determination of the vertical structure of the hydrometeors above the ACRF sites (Clothiaux et al., 2000). Besides mapping the vertical structure of clouds and precipitation at the ACRF sites, the MMCR Doppler moments form the basis of a number of algorithms for retrieving cloud microphysical and radiative properties.

The ACRF MMCRs are vertically pointing ground-based Doppler systems, designed for long-term, unattended operations. Despite their low peak power $(100 \mathrm{~W})$ they have excellent sensitivity, sufficient for detecting most visible clouds overhead. This is achieved through their short wavelength (the backscattering cross section of cloud droplets is $42 \mathrm{~dB}$ stronger at $35-\mathrm{GHz}$ than at 3-GHz, a typical weather radar frequency), the use of a large antenna, long sampling duration and pulse compression techniques. The MMCRs have now been operating at the ACRF sites for several years (i.e., since 8 November 1996, 25 March 1998, 22 October 1998, 14 June 1999 and 6 March 2002 at the Southern Great Plains [SGP]; North Slope of Alaska [NSA]; Tropical Western Pacific [TWP]-Nauru, Manus and Darwin sites, respectively).

The recent development of new sites (e.g., Darwin, ARM Mobile Facility [AMF]) generated the need for the acquisition of additional radars, either exact duplicates of the current deployed MMCR (e.g., Darwin), or new radars (Mead et al., 2005) operating at higher frequency (95-GHz, AMF). This report summarizes the current hardware and software status of the ACRF MMCR utilizing the current state-of-the-art available technology for millimeter wavelength Doppler radars. The newly acquired 95-GHz radars will not be the focus of this review, since they were recently developed and thus integrate in their design several new technological advantages such as digital receiver, high dynamic range, and polarization. Furthermore, preliminary comparison of the 95-GHz Doppler radar with the MMCR at the SGP site demonstrate that the $95-\mathrm{GHz}$ has excellent sensitivity, suppresses the clear-air clutter from insects, and is highly sensitive to weak returns from boundary layer clouds and cirrus layers.

\subsection{MMCR Technical Specifications}

The ACRF MMCR transmitter is a high-gain amplifier with excellent high-phase stability and lowamplitude ripple across the pulsed radio frequency (RF) output. Peak RF power is $100 \mathrm{~W}$ with a maximum duty cycle of $25 \%$ for use with long pulse width, phase-coded waveforms. The tube is run just under saturation and has several RF test ports used for monitoring the transmitted pulse for calibration. The modulator consists of a pulse amplitude modulator and a binary phase modulator used for pulse coding. The intermediate frequency output signal is $60 \mathrm{MHz}$ at $+10 \mathrm{dBm}$. The receiver has a digitally 
controlled blanking circuit, a high gain intermediate frequency (IF) amplifier and separate I and Q baseband channels with four computer selectable bandwidths. Each bandwidth has a separate analog gain control. The blanking circuits protect the receiver and prevent the transient signals that are present near the transmit pulse from causing ringing in the receiver's Bessel filters. Isolation is better than $70 \mathrm{~dB}$. The IF and receiver amplifiers have a combined gain of about $72 \mathrm{~dB}$ with a noise figure of less than $12 \mathrm{~dB}$. The $60-\mathrm{MHz}$ IF input signal is converted to the 34.86-GHz RF output signal through a series of heterodyne mixers. The up converter uses a double heterodyne converter with local oscillator frequencies of 2.0 and $32.8 \mathrm{GHz}$. These are generated from a 100-MHz phase locked signal source with output frequencies of 2 and $16.4 \mathrm{GHz}$. A frequency 2x multiplier generates the 32.8-GHz signal. A front panel attenuator for TWTA saturation control (up to $7 \mathrm{dBm}$ ) sets the RF output level. The 2-GHz IF signal can be directed through an external delay line for receiver delay (range) calibration. The down converter uses the same IF frequencies to mix down to the $60-\mathrm{MHz}$ output. The down conversion process eliminates the unused side band noise. The total gain is about $7 \mathrm{~dB}$ and the noise figure is about $5 \mathrm{~dB}$. All RF signals use WR-28 waveguide.

The need for continuous and robust operation leads to the decision to use the digital signal processors (DSPs) used on the National Oceanic and Atmospheric Administration (NOAA) wind profiler network. This Profiler On-line Program (POP) software that runs under OS/2 environment provided the necessary robust and continuous operational stability required for the collection of long record of clouds observations at the ACRF sites. Recently, a two-stage upgrade (first C-40 DSP-based processors and later the PIRAQ-III digital receiver) of the MMCR signal-processing units was completed. These changes result in higher temporal sampling rate (factor of 4), and continuous recording of the radar Doppler spectra - information that could help to improve the radar data quality control, alleviate issues related to clutter and radar artifacts, and improve our capability to retrieve cloud microphysical and dynamical information. The conclusions from a recent review of the signal processing and sampling strategy of the MMCR during the use of the POP software (Kollias et al., 2005) were used to assess the current status of the MMCR signal processing capabilities. Furthermore, the acquisition of new radars operating at different frequencies and having scanning capabilities that could enhance the information extracted by the existing radars at the ACRF sites is discussed.

\section{Current State of Millimeter Radar Technology}

Recent advances in millimeter-wave radar component technology have led to the development of a new generation of radars above $30 \mathrm{GHz}$. These radars are more reliable and more versatile than previous millimeter-wave radars and consequently are gaining popularity among the remote sensing community, particularly among atmospheric scientists. Since 1990, at least 30 millimeter wave cloud radars have been built worldwide for atmospheric science applications. Many of these radars operate near $35 \mathrm{GHz}$, where atmospheric attenuation due to water vapor and liquid water is considerably reduced as compared to attenuation near $95 \mathrm{GHz}$. Cloud radars operating at $35 \mathrm{GHz}$ generally employ comparatively energetic transmitters (10-100 W average power) and large antennas (1-3 meters diameter). $95 \mathrm{GHz}$ cloud radars, which benefit from the increase in small droplet scattering efficiency, typically operate with less average power ( 0.1 to $15 \mathrm{~W}$ ) and smaller antennas ( 0.3 to $2 \mathrm{~m}$ diameter). The most sensitive systems can detect clouds with reflectivities of $-50 \mathrm{dBZ}$ at the several-km range. 
Pulse compression also offers more affordable access to coherent transmitter technology because pulse compression transmitters are low peak power (such as the TWT transmitter at the ACRF MMCR) and thus less expensive than their more traditional klystron counterparts. The basis behind pulse compression is that if a pulsed waveform is transmitted that contains excess bandwidth given the length of the pulse, a receive filter can be constructed such that targets within the pulse volume can be resolved. This is in contrast to pulsed radar of no excess bandwidth in which the resolution of such targets would not be possible. In other words, as stated above, the combination of the sensitivity gain of the long pulse and the fine range resolution of the short pulse can be realized. There are several different techniques to increase the bandwidth of a pulse. The MMCR employs the phase coding technique within a pulse using coding techniques such as Barker Codes (Mudukotore et al., 1988). Although practicable and relatively easily realized, serious limitations apply and will be discussed in the next section. Examples of higher performance pulse compression techniques are the linear and non-linear frequency modulation (LFM and NLFM) of the pulse.

The use of digital receivers is another emerging development that can improve the performance and flexibility of millimeter wavelength radars. By applying direct intermediate frequency-to-digital conversion (IF sampling) and direct digital signal synthesis (DDS), digital radar receivers can be designed. Digital radar receivers are the trend of the future. Such digital receiver/transmitter modules are already available for Doppler weather radars, and recently the National Weather Service (NWS) decided to upgrade the U.S. network of $10-\mathrm{cm}$ WSR-88D weather radars with digital receivers. Millimeter wavelength radars have higher operational Pulse Repetition Frequency $(10-20 \mathrm{kHz}$ for cloud radars, $0.5-2 \mathrm{kHz}$ for weather radars), higher range resolution (15-45 m for cloud radars, $100-250 \mathrm{~m}$ for weather radars), and require Doppler spectra recording and processing.

Various research groups have been involved lately in the development of such digital receivers suitable for millimeter radars. The newly renamed NOAA Physical Science Division (previously known as ETL) has been involved recently in the development of digital receivers. Engineers in the National Center for Atmospheric Research (NCAR, the same group that was involved in the development of the PIRAQ digital receivers for wind profilers) are involved in the development of a high performance digital receiver/transmitter for the HIAPER 94-GHz radar. In addition, the National Aeronautics and Space Administration (NASA) ER-2 research group is working towards the development of a digital receiver/transmitter for their TWT-based UAV X-band radar. These isolated efforts from various atmospheric research groups verify the trend toward digital receiver/transmitter technology for radars. Furthermore, it illustrates the lack of an off-the-shelf available digital receiver/transmitter hardware and software for millimeter radars. The recently developed ACRF 95-GHz Doppler radars for the SGP and ARM Mobile Facility (AMF) sites include a digital receiver/transmitter. It is important to mention that the implementation of a high performance pulse compression scheme is directly coupled to the presence of a digital receiver/transmitter in the radar. 


\section{ACRF MMCR Current Status}

\subsection{RF Components}

The Traveling Wave Tube (TWT) technology, the frequency (35-GHz), the RF electronics, antenna, the calibration and radar control circuits and the environmental enclosure of the MMCR remain assets for our ability to detect the profile of hydrometeors at all heights in the troposphere at the ACRF sites.

\subsection{MMCR Receiver}

\subsubsection{NOAA Profiler On-line Program (POP)}

On the MMCR receiver side, we have ongoing DSP upgrades. From the early days of MMCR operations the need for continuous and robust operation leads to the decision to use the digital signal processors (DSPs) used on the NOAA wind profiler network and the POP software that run under OS/2 environment. The POP is the basic wind profiler radar processor, and robust operation at remote locations was more important than high temporal resolution. Thus the processor efficiency was very poor (5-30\%) and Doppler spectra recording was not available. Cloud researchers, however, are more interested in shorter time scales (Kollias et al., 2005). However, since the early days of the MMCR development, the NOAA Environmental Technology Laboratory (ETL) through a Cooperative Research and Development Agreement (CRADA) transferred the MMCR technology to Radian, Inc.

\subsubsection{C-40 DSP Board}

With support from ACRF and NOAA, Radian began the design of a new DSP board for the MMCR. The new board (C-40 DSP from Texas Instruments) is a 5 DSP-on-the-board processor. The advantages of the new design are the use of higher clock frequencies and multiple DSPs working in parallel to accelerate the radar's processing power. The hardware runs under a new radar control software program (LAP-XM) under Windows 2000. There were significant delays but NOAA/ETL completed with Radian work and the upgrade was finally installed at the SGP and NSA site. This processor also is installed on the NOAA MMCR now located in Eureka, Canada. The processor efficiency of the new board is 50\%. Preliminary analysis of Doppler spectra recorded by the C-40 boards, illustrated the presence of spectral image when the peak spectral power is higher than 30-35 dB above the noise. This feature is a nuisance to radar meteorologists and scientists that use the Doppler spectra for microphysical retrievals. However, Texas Instruments manufactured only three A/D C-40 boards before the C-40 processor board was slated for discontinuity. Reverse engineering of the board or a new replacement board required significant engineering and software development. As a result, only the SGP and NSA sites were upgraded with the C-40 processor.

\subsubsection{Vaisala Inc. PIRAQ-III Digital Receiver}

At the same time period, NCAR developed a PC-Integrated Radar Data Acquisition System (PIRAQ) and Vaisala Inc. was licensed by NCAR to use the technology for its wind profiler systems with PIRAQ-III boards running under LAP-XM (Radian Inc. was bought by Vaisala Inc.). The new PIRAQ-III was selected for upgrading the TWP sites. The benefit of the PIRAQ-III is that the boards and software are supported products of Vaisala (Widener et al., 2004). The processor efficiency of the PIRAQ-III is close 
to $70 \%$, an improvement over the C-40 processors. The PIRAQ-III cannot support pulse compression and cannot be used for the recording of I/Q data. An evaluation of the performance of the PIRAQ-III is not completed yet at this time, since the first deployment of the system (TWP-Darwin) took place in November 2005 just before the Tropical Warm Pool-International Cloud Experiment (TWP-ICE). However, it is a signal processing unit design for wind profilers (processor efficiency $70 \%$, that cannot be coupled to the transmitter unit and cannot accommodate complex waveforms that could improve the sensitivity of the MMCR.

\subsubsection{Present Status of MMCR Receivers}

As of December 2005, all three signal processing units described above are used by the ACRF MMCRs (Figure 1). The ACRF TWP Nauru and Manus sites have the original receiver (POP) processor. The POP processor produces reliability problems (e.g., OS/2 stability issues as a result of the Y2K upgrade)

\section{ACRF MMCR Signal Processing Unit Upgrades}

\section{Spring 2006}



Figure 1. Past, present and future upgrades of the SCRF MMCR receiver 
along with all the other issues mentioned in the study by Kollias et al., (2005). The SGP and NSA sites have the C-40 processor that is obsolete and produces Doppler spectra image problems that we cannot address due to high engineering cost and lack of hardware components. Finally, the TWP Darwin site has the PIRAQ-III digital processor. The PIRAQ-III receiver was evaluated recently and provides the highest temporal resolution (1.5s), and data processing efficiency (60-70\%). The issue of Doppler spectra images that have troubled scientists in the past with the POP and C-40 processor was also evident in the Doppler spectra collected with the PIRAQ-III at the Darwin site. The source of the presence of Doppler images in the digital receiver was identified and it is expected that in the near future (before the TWP-ICE experiment) the new digital filter implementation will suppress the Doppler image significantly.

\section{ACRF MMCR Future Upgrades}

\subsection{Near-term Plans (0-2 years)}

\subsubsection{Upgrade Manus/Nauru to PIRAQ-III}

The near-term plans for the MMCR receivers include the upgrade of the Manus and Nauru MMCR receivers from the POP processor to the PIRAQ-III receiver. All the necessary parts have been procured and are on site at Darwin. The upgrade is scheduled for early March 2006. The PIRAQ-III digital receiver is supported through Vaisala Inc. and Coy Chanders, a software consultant to NOAA and Vaisala. Coy Chanders along with Ken Moran from NOAA were instrumental in the preparation of the first PIRAQ-III system that is currently deployed in Darwin.

\subsubsection{Upgrade SGP/NSA to PIRAQ-III}

In 2006, a decision is expected on the upgrade of the remaining ACRF sites (SGP and NSA) with PIRAQ-III digital receivers. Although the evaluation of the PIRAQ-III digital receiver is not completed yet, preliminary analysis of observations from the TWP Darwin site demonstrate that the PIRAQ-III receiver has higher efficiency, and it is expected that its easy-to-change software $(\mathrm{C} / \mathrm{C}++)$ can be adjusted to suppress the Doppler image that also was present in the TWP Darwin MMCR Doppler spectra. Furthermore, the SGP and NSA MMCR radars currently have a signal processing unit (C-40) that is obsolete and difficult to reprogram in order to eliminate or suppress the Doppler spectra image problem. Furthermore, there are no spares if a C40 board should fail. If this were to happen, that particular MMCR would be down until the board was repaired (if possible) or the radar was upgraded. The upgrade (scheduled for early 2007) will bring commonality among the five operational MMCRs at the ACRF sites, reduce the maintenance costs, and provide uniform temporal resolution and radar operational modes in all the ACRF sites. Each signal processing unit upgrade is estimated to $\sim \$ 30 \mathrm{~K}$ per MMCR.

\subsection{Long-term Plans (2-5 years)}

\subsubsection{Digitally Generated Pulse Compression}

Despite the use of a high duty cycle TWT transmitter, the use of pulse compression or alternatively the use of the high duty cycle of the MMCR TWT transmitter is very limited. The phase-coding pulse compression scheme currently used in the MMCR provides $-30 \mathrm{~dB}$ peak side lobes and adds high overhead in the signal processing time. In the early 90s, the phase-coding pulse compression scheme was 
relatively simple to implement with the use of analog binary phase modulator. The current analog-based pulse compression MMCR hardware is not capable of generating other sophisticated pulse waveforms with superior peak side lobe suppression (e.g., Non-linear FM [NLFM] pulse compression schemes can generate $-60 \mathrm{~dB}$ peak side lobes). Recently, the use of pulse compression was eliminated from the $\mathrm{BL}$ mode (Kollias et al., 2006). The MMCR cirrus mode (Clothiaux et al., 2000) is currently the only MMCR operational mode that utilizes pulse compression. Thus, although the TWT is an amplifier suitable for pulse compression, this not manifested in the case of the MMCR. If we use a digitally generated IF pulse waveform with embedded NLFM or other pulse compression schemes, we could achieve better range side lobe filtering and higher performance. When the signal is digitally constructed at the lower IF ( $60 \mathrm{MHz}$ for most millimeter wavelength radar), complex waveforms can be generated and fast switching is available (e.g., frequency hopping systems). In addition, we can eliminate the restricted precision and distortion of analog detectors that greatly limit the improvement of the performance of the MMCR. The use of a PC-based digital-to-analog (D/A) card for the direct digital synthesis (DDS) of the IF transmitter waveform will result to low maintenance cost and further upgrades will be limited only to upgrades of the PC computing power.

\subsubsection{SBIR Development for a New Digital Transceiver}

The development of a modular FPGA-based digital receiver/transmitter for the ACRF profiling cloud radars could lead to superior performance, $100 \%$ processor efficiency, coupling between the DDS and the digital receiver, and will allow the use of pulse compression. The digital radar receiver directly samples the IF signal of the received channels prior to detection. All subsequent processing and detection are accomplished in the digital domain. By utilizing these novel digital techniques, the digital radar receiver provides persuasive advantages that are difficult or impossible to achieve with an analog receiver. These advantages include processing accuracy, flexible interface with the radar control unit, convenient control of transmitter waveforms and LO frequency of the receiver and higher stability (no temperature sensitivity and drifts). Some of the basic characteristics of this FPGA-based digital receiver front end will be 1) operate at high PRF (10-20 kHz), 2) provide flexible programmable IF front end (e.g., FIR filter, demodulation), 3) provide high range resolution (e.g., $15 \mathrm{~m}$ ), 4) provide Doppler spectra for profiling radars, and 5) moments (dBZ, Kdp etc) for scanning mode.

\subsubsection{Development Challenges}

The development, design, and testing of future MMCR upgrades such as the digital transceiver brings important development challenges. In the past, NOAA originally had a hot spare MMCR (it didn't include the antenna) paid for by DOE. However, during various upgrades, the "hot spare" became the spare parts depot for the other MMCRs. It was not kept up to date. Parallel with the DOE hot-spare, NOAA developed a MMCR as part of their on-going research activities. As part of the Study of Environmental Arctic Change (SEARCH) Program, NOAA deployed the hot-spare MMCR at a climate monitoring site in Eureka, Canada, in the summer of 2005. The NOAA hot-spare MMCR was used as a test bed for the development and testing of past MMCR upgrades, such as the C-40 signal processing unit. In addition, laboratory tests and preparation of the PIRAQ-III prior to its first deployment at the Darwin site were completed at the NOAA/ETL using RF lab testing equipment. 
Currently, the ACRF has no hot-spare MMCR that can be used for the development and testing of the next generation digital transceiver. In addition to the lack of a hot-spare MMCR, the ACRF has no "inhouse" laboratory infrastructure and technical expertise to conduct tests, evaluation, and development of present and future MMCR components The development of inexpensive X-band radar with similar IF and timing requirements with the MMCR in the context of the proposed SBIR for the development of the next generation digital transceiver can be another solution for the development of ACRF test bed radar.

\subsubsection{MMCR Antenna Refurbishment}

The MMCR antenna is a fixed position, vertically pointing parabolic dish with a rear-mounted feed and Cassegrain sub-reflector. A 10-foot diameter antenna, which has a gain of $57.2 \mathrm{~dB}$ and a beam width of $0.19^{\circ}$, is used on the SGP radar. The smaller 6-foot-diameter antennas have a gain of about $53.4 \mathrm{~dB}$ and a beam width of $0.29^{\circ}$ and are deployed at the NSA and TWP sites. The MMCR antennas are original MMCR hardware. Recently, we evaluated the polarization isolation of the SGP antenna and it was found poor. Furthermore, the TWP sites antennas are showing signs of corrosion. We suggested that in the next 2-3 years we initiate an effort to refurbish the MMCR antennas. The MMCR antenna refurbishing is a time-consuming effort that involved extensive antenna transportation time from the remote sites to the refurbishing location. After refurbishment, the antennas must be recharacterized at an antenna test range to determine their gain and beamwidths. We need to purchase a spare antenna (6-foot diameter, $\$ 50 \mathrm{~K}$ ) that will rotate through the ACRF site and replace the antenna that will be sent for refurbishing. This will allow us to minimize the radar down-time during this process. The estimated refurbishing cost per antenna is $\sim \$ 30 \mathrm{~K}$ (SGP more). The MMCR antenna refurbishing project could start after we have completed the PIRAQ-III upgrades at the SGP and NSA sites and thus spread the financial burden over several years.

\subsubsection{Operational Concerns}

A complete migration path is required not only on the technological aspects of the MMCR upgrades but also for the development of testing and diagnostic capabilities from the site engineers and the MMCR mentors in order to ensure that the MMCR will continue to have high reliability, uptime, and superior sensitivity. The limited technical capabilities at the ACRF sites prevent strong intervention in the MMCR hardware/software by the locals. Thus, it is important that we have a continuous and consistent monitoring of the ACRF MMCR health status and data quality.

The continuous recording of the Doppler spectra by the ACRF MMCR and the W-band radars at the SGP and AMF sites generates large data volumes $(600 \mathrm{MB} / \mathrm{hr}$ at the TWP Darwin site) that currently are transferred with plug n' play hard disk drives that are shipped back and forth from the remote sites. The proposed on-site cloud masking will result in a significant reduction of the data volume (80-90\%). Even at this reduced MMCR data volume generation, the current bandwidth limitations at the remote TWP /NSA sites make difficult if not impossible the data transfer via the internet. We should look into possible upgrades of the bandwidth at the TWP/NSA sites that could accommodate these data rates. The MMCR mentor should schedule annual site visits for calibration inspection, enhance work with the MMCR sites, and develop algorithms for ascertaining trends related to the operational status of the MMCR. Currently, the mentor is under funded to do the aforementioned tasks. 
Finally, the advancements in radar meteorology technology such as the use of sophisticated pulse waveforms, digital receivers, and phased-array antennas is transforming the field of radar meteorology and introduces advance concepts in digital signal processing, pulse compression, and other topics. ARM scientists must maintain contact with this new, low cost, and powerful radar technology, since it can lead to the development of new sophisticated and innovative radar tools that can address particular scientific objectives such the 3-D mapping of the cloud field or high resolution cloud imaging.

\section{Path Forward for the Acquisition of New ACRF Radars}

Radar applications should look beyond the use of sensitive cloud profiling radars such as the MMCR's at the ACRF sites. Continuous technological developments (e.g., reliable power supplies for high frequency transmitters/amplifiers, off-the-self high quality RF components, digital IF receivers, and phased-array antennas), the use of new platforms (e.g., UAV), the diversity of cloud and precipitation conditions, and the universal need to achieve higher resolution in 3-D will lead to the need for developing new active sensors and radars in particular. Some new suggested radars systems are discussed in the following subsections.

\subsection{X-band Vertically Profiling Radar (X-VPR)}

$\mathrm{X}$-band frequency is the most desirable for the development of relatively inexpensive (around $\$ 200 \mathrm{~K}$ ) vertically profiling cloud and precipitation radars since high power transmitters and off-the-shelf components are readily available and thus costs are relatively low in comparison with other higher frequency radars. Such a system can provide good sensitivity ( $-25 \mathrm{dBZ}$ at $10-\mathrm{km}$ range) and the attenuation problem in heavy rain is tolerable in a vertically pointing mode. The X-VPR system will have no scanning and polarimetric capability and could be host in a container. The design of the X-VPR is based on the concept to develop inexpensive radar using as much as possible off-the-shelf components to reduce costs without compromising the performance of the system. The development of additional XVPR units should be minimized once the first system is designed.

The X-VPR system utilizes a modified version of low-cost; magnetron-based marine radar transceiver, which can be upgraded with an improved local oscillator and receiver front-end. A 1.8-m diameter parabolic antenna will provide a $1.5^{\circ}$ degree beam width and $42 \mathrm{~dB}$ gain. The temporal resolution will be $2 \mathrm{sec}$ and the range resolution will vary from 45 to $90 \mathrm{~m}$. Such systems have been developed by the University of Miami (Figure 2), McGill University (Figure 3) and recently by the NASA Goddard Space Flight Center for use in high altitude UAVs (Li et al., 2005). The receiver will be a single conversion design, with off-the-shelf radar IF front end (low noise amplifier, down conversion chain and STALO) and a digital receiver that will sample both the received atmospheric signal (signal IF) and a sample of the transmitter pulse (reference IF) for Doppler capability. The digital receiver will be based on a compact PCI (cPCI) Linux-based PC system and will be produce Doppler moments and spectra. Inexpensive and low-maintenance cost X-VPR systems can be deployed at the ACRF sites and improve our ability to sample clouds and precipitation. The X-VPR Doppler moments can be used for the development of dualwavelength (X/K-band) based retrieval techniques for cirrus and precipitating clouds. Parameters such as the differential reflectivity ratio (DZR) and differential mean Doppler velocity (DDV) can be used as input in microphysical retrieval techniques. Furthermore, the X-VPR has sufficient sensitivity to sample non-precipitating clouds during periods that the ACRF MMCR is down. 


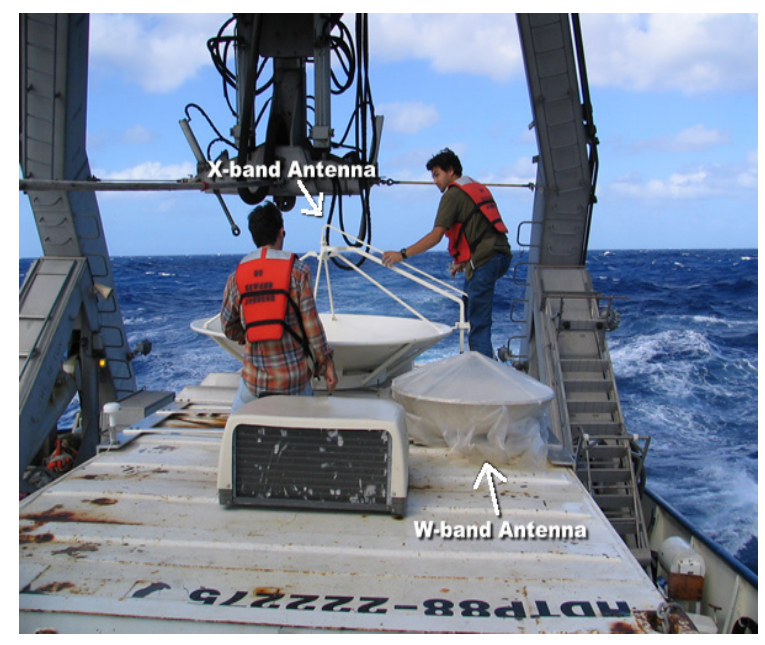

Figure 2. The University of Miami X-band VPR radar

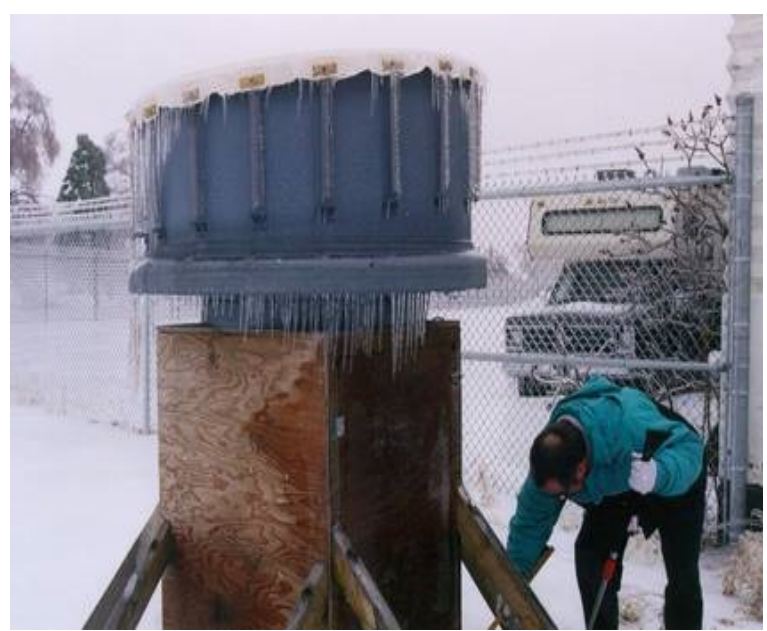

Figure 3. The McGill University X-band

\subsection{Dual Polarization Scanning X-band Cloud Radar (DPS-X)}

X-band radars (9.4-GHz frequency) have recently received much attention due to their capability of detecting useable $\mathrm{K}_{\mathrm{DP}}$ magnitudes in rain rates as low as $\sim 2 \mathrm{~mm} \mathrm{hr}^{-1}$, while useable $\mathrm{S}$-Band $\mathrm{K}_{\mathrm{DP}}$ data cannot be obtained at rain rates of approximately 5-7 $\mathrm{mm} \mathrm{hr}^{-1}$. X-Band $\mathrm{K}_{\mathrm{DP}}$ estimators are thus able to perform over a significantly larger portion of raining area and extend polarimetric rain estimation capabilities lower into the rain rate distribution than those at S-Band, which allow improvement in rain estimates in rain rate estimates over traditional Z-R techniques (Rutledge et al., 2005). Ideally, collocated dual wavelength radar measurements of rainfall can combine the advantages of both bands, i.e., the availability of polarimetric differential phase estimates for lighter rainfalls at X-band and longer-range coverage with negligible attenuation at S-band (Matrosov et al., 2005). One of the instrumentation recommendations from the recent Global Precipitation Mission (GPM) - Ground Validation (GV) pilot study (Rutledge et al., 2005) for the Super site is the operation of dual wavelength (S- and X-band) scanning dual polarization Doppler radar.

The DPS-X system should be transportable, scanning, and Doppler. Simultaneous HV mode transmission that will result in pairs of pulses with equal power simultaneously in horizontal and vertical polarizations is desirable. This will result in linearly polarized waves with a 45-degree tilt. This is the same scheme recommended for future polarization upgrades to NEXRAD (Doviak et al. 2000). Polarization parameters from this mode include differential reflectivity $\left(Z_{\mathrm{DR}}\right)$, propagation differential phase shift $\left(\phi_{\mathrm{DP}}\right)$ and its range derivative, specific differential phase $\left(\mathrm{K}_{\mathrm{DP}}\right)$, and polarization correlation magnitude at zero lag $\left(\rho_{\mathrm{HV}}\right)$. These polarimetric parameters are used to estimate precipitation rates and/or hydrometeor types.

A 3-m diameter antenna will provide a $1^{-}$degree beam width and $44 \mathrm{~dB}$ gain. A 9.4-GHz TWT amplifier with $5 \%$ duty cycle and $25 \mathrm{~kW}$ peak transmit power will provide sufficient sensitivity for the detection of clouds at long range (required sensitivity $-25 \mathrm{~dB}$ at $20 \mathrm{~km}$ range). It is critical to achieve such superb sensitivity with the DPS-X. This will allow the DPS-X to serve as a cloud and precipitation radar. The 
excess sensitivity (compared with previous similar systems) is provided by the use of pulse compression. The high sensitivity of the DPS-X will allow the mapping of the $3 \mathrm{D}$ cloud structure at long ranges in the absence of rain.

A digital receiver with technical specifications and performance analogous to RVP8 (the digital receiver selected for the WSR-88D upgrade (Ohara et al., 2005)) that includes the Digital Receiver, Digital Waveform Generator Card, and all software including pulse compression, realtime I/Q archiving/playback, full scientific source code and development environment and also supports polarimetric radars. NOAA/ETL (currently ESRL Physical Science Division (PSD)) has long operated a pair of transportable X-band Doppler radars for observing storm kinematics, boundary layer airflow, ocean surface features and tracking air parcels with chaff. One of these radars was outfitted in 1985 for circular dual-polarization measurements. More recently, that radar has been retired while the other, named NOAA/D (Figure 4), has become the focus of extensive hardware and data system upgrades (Martner et al., 2001). Recently, the NOAA/K scanning radar was put out of commission on October 31, 2005, and ESRL/PSD plans to use the missile tracking scanner of the NOAA/K to develop an X-band scanning radar (for the Department of Defense), and eventually in the future plan to develop a system similar to DPS-X as part of the GPM GV requirements (T. Schneider and Brooks Martner, ESRL/PSD, personal communication, 2005).

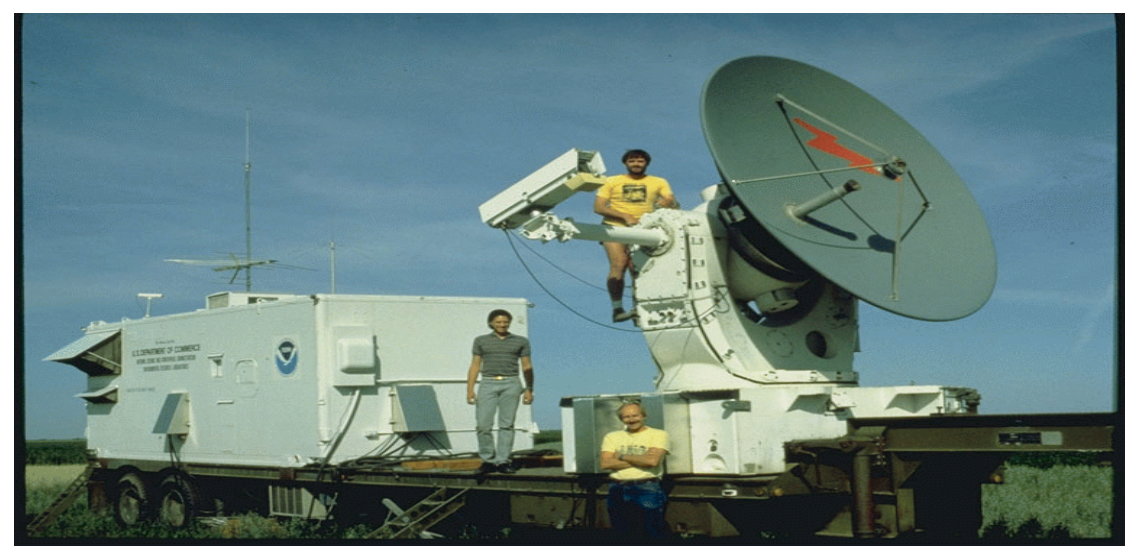

Figure 4. The NOAA/D X-band scanning and polarimetric radar

\subsection{3-D Volume Imaging Radar (3D-VIR) - System Concept}

This radar system will address the need for mapping the 3D cloud structure (Spatial/3D CPWG Focus Group report) over an extensive area (40x40x20 km volume box - "local scale") around the ACRF SGP central facility. This can be achieved either with a single Doppler radar system or a combination of radar systems (e.g., an array of networked scanning radars). Here the concept of a multi-functioning 3D Volume Imaging Radar (3D-VIR) is developed. The system concept of the 3D-VIR will explore the use of new emerging radar technologies such as the use of phased array antennas and the use of pulse compression that can add agility and multi-purpose design to the 3D-VIR (Figure 5). 


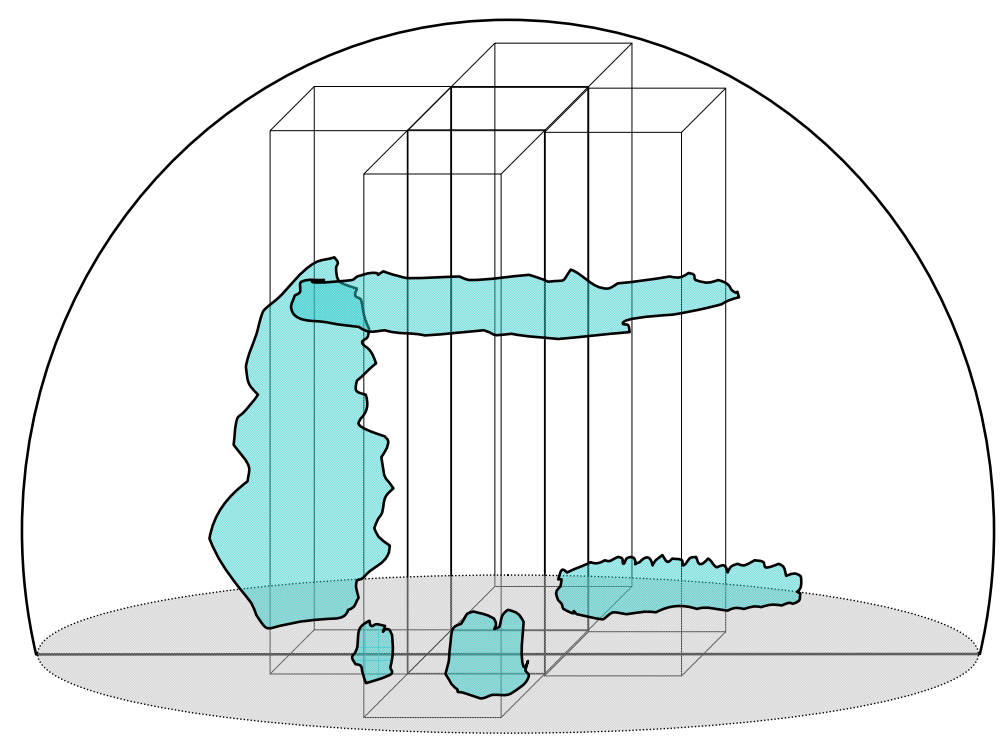

3D Volume Imaging Radar (3D-VIR)

Surveillance Mode.

Surveillance box Hemispherical Scanning

Sensitivity at boundary $40 \times 40 \times 15 \mathrm{~km}$

Range Resolution... $-20 \mathrm{dBZ}$

Temporal Resolution $60 \mathrm{~m}$

Products

$1 \mathrm{~min}$

3D Volume Imaging Radar (3D-VIR)

IOP Mode

Range Resolution.

Temporal Resolution.

Products.

Sector Scanning $30 \mathrm{~m}$

$10 \mathrm{sec}$

Raw I/Q, Doppler
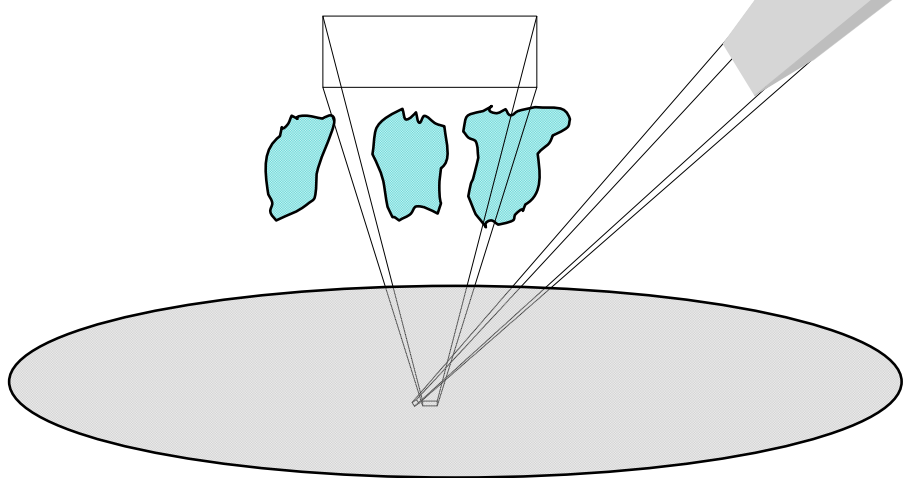

Figure 5. The operational modes of the 3D-VIR system 
Spacing requirements between the active antenna elements and cost suggest the use of a 9.4-GHz TWT amplifier with $5 \%$ duty cycle and $25 \mathrm{~kW}$ peak transmit power. The $2 \times 2 \mathrm{~m}$ phased array antenna will provide a 1.25 degree beam width, 25 elevation beams with pulse-to-pulse frequency hopping, 10 degrees per second azimuth scan rate, and $0^{\circ}$ to $60^{\circ}$ elevation scan. The antenna could be tipped mechanically in elevation to cover the conical section near nadir. With the use of 20:1 pulse compression scheme and $60-\mathrm{m}$ range resolution, the radar should be able to obtain- $20 \mathrm{dBZ}$ sensitivity at $20 \mathrm{~km}$, ignoring rain attenuation. The rapid scan capability of this radar system presents new challenges with regard to calibration, clutter removal, radar control, maintenance, and data processing and visualization.

Active electronically scanning, phased-array radars are in the center of future advance radar technologies. The MIT Lincoln Laboratory (Weber et al., 2005) have recently begun exploratory research on the capabilities and technology issues related to the use of multi-function phased array radar (MPAR) as a possible replacement approach for the 10-cm wavelength NEXRAD or WSR88-D (Serafin and Wilson, 2000) national-scale weather radar network. This is managed jointly by the NWS, the Federal Aviation Administration (FAA), and the Department of Defense (DoD).

In addition, the National Severe Storm Laboratory (NSSL) is evaluating (Forsyth et al., 2005) the use of The National Weather Radar Testbed (NWRT), a10-cm phased array radar (Figure 6) for use in studying and developing faster and more accurate warning, analysis and forecast techniques for severe and hazardous weather and testing multifunction capabilities such as aircraft tracking, wind profiling and weather detection at the same time. Finally, the Naval Post Graduate School's Center for Interdisciplinary Remotely Piloted Aircraft Studies (CIRPAS) in collaboration with ProSensing Inc., recently modified a phased array X-band tactical radar system to add a weather surveillance mode (PopStefanija et al., 2005). The modified system, reconfigured for mobile operation from a heavy-duty truck, is shown in Figure 7.

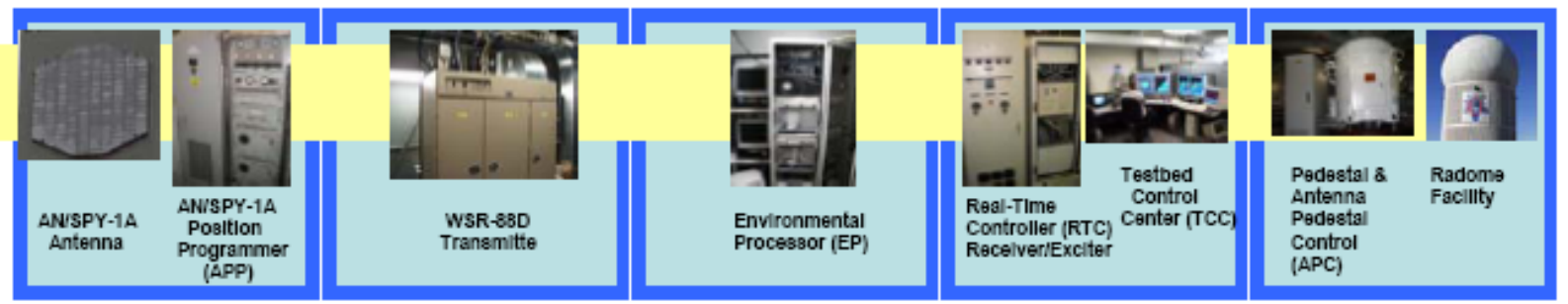

Figure 6. The National Weather Radar Testbed (NWRT) Equipment

The basic function of the proposed system will be a 3D cloud and precipitation surveillance mode (Figure 5). 3D cloud and precipitation mapping over a large volume is of great importance in order to address scientific issues related to the radiative effects of 3D clouds and the representation of clouds and precipitation over several grid box of cloud resolving models (CRMs). Furthermore, this 3D mapping of clouds and precipitation will present new opportunities for satellite radiance and retrieval (e.g., microphysics, multi-layer) validation and comparison with the ground-based sites. The coverage area should be a $40 \times 40 \mathrm{~km}$ box around the central facility. The required sensitivity in the boundary of the surveillance box is $-20 \mathrm{dBZ}$ (at fast surveillance scanning mode sampling conditions). Operational radar observables will be the Doppler moments (Pulse-Pair algorithm). In addition, the full volume scan should be completed within $60 \mathrm{sec}$. 


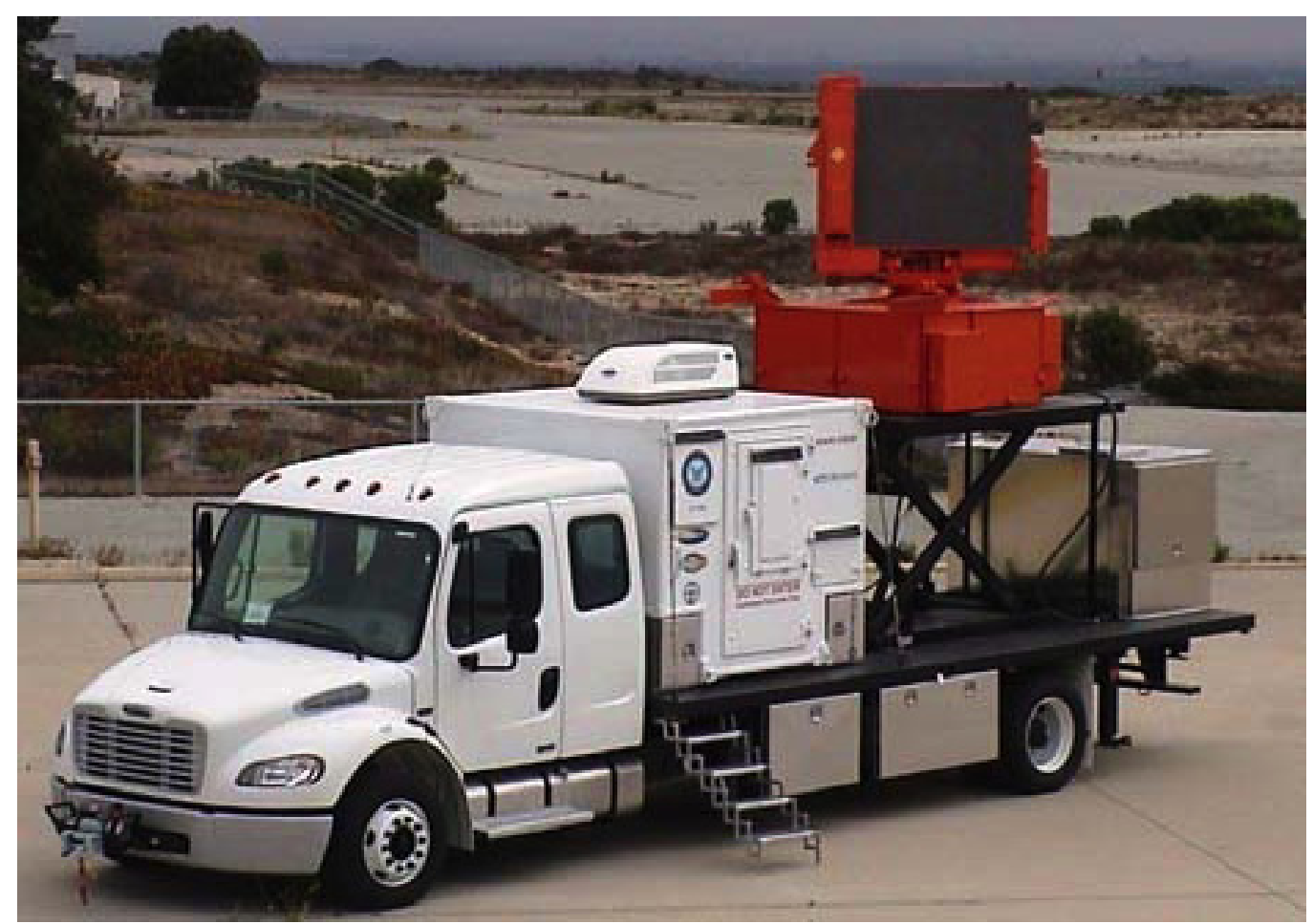

Figure 7. MWR-05XP installed on a heavy-duty truck. The $1.2 \mathrm{~m}$ phased array antenna provides electronic beam scanning in elevation and combines electronic back-scanning with mechanical scanning in azimuth.

In addition to the surveillance mode, 3D-VIR will be used during ARM field campaigns to address campaign specific objectives such as rapid small sector off zenith scan of boundary layer clouds or follow cloud entities that enter the sampling box or are sampled by aircrafts. Thus, multipurpose scanning antenna and radar design suitable for a variety of field campaigns are required.

\section{ARM - Unmanned Aerospace Vehicle (UAV) Cloud Radar}

\subsection{Background}

The U.S. Department of Energy Atmospheric Radiation Measurement Unmanned Aerospace Vehicle (ARM-UAV) program was initiated as part of the larger DOE objective of improved understanding of the role of clouds in global climate change. Most of the science objectives related to the ARM-UAV program require the presence of airborne cloud radar for the retrieval of cloud boundaries and microphysical properties throughout the entire cloud column. In the late 1990s, DOE partnered with the University of Massachusetts Microwave Remote Sensing Laboratory (UMass-MIRSL) for the development of a solidstate 95-GHz airborne cloud millimeter wave radar. This compact Millimeter-wave Radar (CMR) flew on the NASA Proteus during a series of engineering and science flights organized by the ARM-UAV program, and it was tested in the ground at the ACRF SGP site next to the ACRF MMCR and also flew the CMR with the ACR on the DOE Twin Otter during the Monterey maritime boundary layer flights in 1999. The CMR had several innovative RF and digital technologies that offered many potential advantages in size and weight; however, this approach has not proven to be reliable in two ARM-UAV 
field deployments undertaken in 2002 and 2004. A review of the status and performance of this radar was undertaken in November 2004 at Denver, Colorado, involving representatives of the ARM-UAV program, DOE, and radar experts from several universities. The consensus of this meeting was that the current approach is not sufficiently developed to provide a reliable cloud radar. Therefore, the ARMUAV program faces a critical need for a new cloud radar based on more proven technologies.

\subsection{UAV Cloud Radar Specifications}

The ARM-UAV program science-based radar requirements favor the use of a compact 94-GHz radar that has excellent sensitivity and narrow beam width with a small antenna. The detailed characteristics of the new cloud radar are:

1. Must be able to operate with minimum of operator intervention for time periods of 8 to 10 hours.

2. Minimum weight (50 kg is a good goal), size and power consumption (not an issue for Proteus but could be issue for future platforms).

3. Must be capable of operating at high altitudes (up to $55 \mathrm{kft}$ ).

4. Required sensitivity is $-35 \mathrm{dBZ}$ at $2 \mathrm{~km} ;-40 \mathrm{dBZ}$ at $1 \mathrm{~km}$ (NOTE: this is a minimum required sensitivity because we begin to lose data in the boundary layer with less sensitivity).

5. Desired vertical resolution is $30-45 \mathrm{~m}$ with $60 \mathrm{~m}$ maximum resolution.

6. Desired horizontal resolution is $100 \mathrm{~m}$ at $200 \mathrm{~m} / \mathrm{sec}$ (implies integration time of $0.5 \mathrm{~s}$ ).

7. Flexibility to change characteristics between flights (pulse width, integration time, range).

8. Down-looking and operating at $94 \mathrm{GHz}$.

9. Required measurement capabilities are reflectivity and Doppler velocity (polarization is secondary requirement).

10. Nyquist velocity should be on order of $20 \mathrm{~m} / \mathrm{s}$ (although $10 \mathrm{~m} / \mathrm{s}$ would be acceptable for cirrus because flying over the intense updrafts with Proteus is not a typical mission)

11. Need to avoid pulse compression in order to profile whole troposphere.

12. Should not create RF or other interference because of proximity to other payloads.

13. Collect spectra to allow for post-processing of Doppler moments after flight; however, the capability for fast download of data after flight is needed so that we are ready for rapid turnaround.

14. Lots of storage capacity for data (to be able to get at noise floor as function of time).

15. Design should provide reliable radar, even if this reduces number of added features.

16. Interface between internal components and antenna should be flexible to allow for mounting on alternate aircraft platforms.

17. Antenna size preferably between 12 inches and 2 feet (or 3 feet, if possible, for increased sensitivity) 


\subsection{Other Airborne Cloud Radar Projects}

The ARM-UAV program should follow closely and interact with other agencies that currently develop sophisticated airborne radar systems. Such efforts include:

- NCAR's plans to develop a 94-GHz cloud radar for their High-performance Instrumented Airborne Platform for Environmental Research (HIAPER), an advanced airborne research platform, currently being built and modified to serve the National Science Foundation's (NSF) environmental research needs for the next several decades. The new aircraft will be maintained and operated for the NSF by the National Center for Atmospheric Research (NCAR) in Boulder, Colorado.

- NASA's future plans for the ER-2 and Global Hawk programs and their efforts to develop inexpensive airborne X-band radars ( $\mathrm{Li}$ et al., 2005).

- The new dual frequency (X- and W-band) radar systems built recently for Canada's National Research Council. The NRC Institute for Aerospace Research (NRC Aerospace), in cooperation with Prosensing Inc., is currently developing a state-of-the-art dual frequency airborne radar system for atmospheric and flight safety research for its Convair-580 research aircraft. The choice of $\mathrm{W}$ - and $\mathrm{X}$-bands for the NRC Airborne radar system is based on suitability for airborne installation (less power and smaller antennas) while providing high resolution and high sensitivity for detection of weak clouds, and the expected research applications of the radar. The $N$ RC $\boldsymbol{A}$ irborne $\boldsymbol{W}$ and $\boldsymbol{X}$-bands radar system (NAWX) will be integrated onto the NRC Convair-580 in December 2005.

These projects incorporate significant R\&D efforts and could provide in the near future (2006) conclusion on the use of pulse compression and high performance digital trans/receivers in high-altitude cloud radars. These R\&D efforts could help to design a radar system for the ARM-UAV system that can be acquired either through the DOE SBIR program or other funding sources. These R\&D efforts suggested that inexpensive X-band radar with TWT transmitter and pulse compression could provide an alternative solution for cloud and precipitation detection if the sensitivity requirements can be adjusted.

\subsection{Path Forward}

The ARM-UAV program has integrated instruments on a variety of UAVs and piloted aircraft and has conducted measurement campaigns in conjunction with ground-, aircraft-, and satellite-based observations. However, the development of suitable cloud radar for these platforms remains a current need.

Although the term UAV refers to an unmanned aerospace vehicle, the Proteus is piloted aircraft and its operational characteristics, space, weight and power consumption requirements are different from that of future UAVs. For airborne cloud radar, the physical space, weight and power limitations are critical to the radar type selection and design (e.g., frequency, antenna, transmitter type). Thus it is important that the characteristics of the ARM-UAV cloud radar are updated to the current and future trends of UAVs.

The initial effort for the acquisition of a UAV cloud radar through the collaboration between the UMassMIRSL and DOE was not successful, and one of the reasons was the lack of sufficient knowledge for the development and design of high-altitude, compact radar systems. Ten years later, the plethora of airborne cloud radar developments from other agencies (e.g., NSF, NASA, and NRC Canada) offers significant 
advancements (e.g., pulse compression, EIK transmitters, digital receivers) in the radar design concepts of these airborne cloud radars that should be incorporated in the design phase of the ARM-UAV cloud radar.

In the near-term, the development of inexpensive X-band radar ( $\$ 300 \mathrm{~K}$ or less) for the ARM-UAV with pulse compression, high temporal and spatial resolution, digital receiver and sensitivity $-35 \mathrm{dBZ}$ at $1 \mathrm{~km}$ range is suggested. This radar can be developed in 2006 with funds from the ARM-UAV program. The radar will be capable of detecting liquid cloud at short distance, and higher reflecting ice and precipitating clouds at any location in the troposphere. This radar can provide a short term solution for the remote sensing of cloud boundaries and microphysical properties and improve substantially the ability of the ARM-UAV to study the radiative properties of clouds.

On the long-term, a W-band cloud radar can be developed through the DOE SBIR program (2009). The design of the W-band radar will incorporate state-of-the-art digital technology. When the W-band development is completed the W-band radar will substitute for the X-band radar. Another interesting future solution would be (if appropriate space and weight conditions are present) to have both radar systems on the ARM-UAV platform. The combination of X- and W-band radar frequencies will provide better microphysical retrievals. If both radars can not be present on the same UAV the X-band can serve as spare radar in case of failure or malfunctioning of the W-band radar.

\section{References}

Doviak, RJ, V Bringi, A Ryzhkov, A Zahrai, and D Zrnic. 2000. "Considerations for polarimetric upgrades to WSR-88D radars." Journal of Atmospheric and Oceanic Technology 17, 257-278.

Forsyth, DE, JF Kimpel, DS Zrnic, R Ferek, JF Heimmer, TJ McNellis, JE Crain, AM Shapiro, RJ Vogt, and W Benner. 2005. "The National Weather Radar Test Bed (Phased-Array)." Preprints, 32th International Conference on Radar Meteorology, American Meteorological Society, Albuquerque, NM.

Li, L, GM Heymsfield, J Knuble, and S Rodriguez. 2005. “A dual-beam X-band Doppler Radar for Tropical Storm Research on High-Altitude UAVs.” Preprints, 32th Inernational Conference on Radar Meteorology, American Meteorological Society, Albuquerque, NM.

Martner, BE, KA Clark, SY Matrosov, WC Campbell, and JS Gibson. 2001. 'NOAA/ETL’s polarization-upgraded X-band "HYDR" radar." Preprints, 30th International. Conference on Radar Meteorology, American Meteorological Society, 101-103.

Matrosov, S, R Cifelli, P Kennedy, SW Nesbitt, VN Bringi, and B Martner. 2005. "On the Role of Xband Radar in extending Longer-Wavelength Radar Polarimetric Retrievals to Lighter Rains." Preprints, 32th International Conference on Radar Meteorology, American Meteorological Society, Albuquerque, NM.

Mead, JB, and K Widener. 2005. "W-band ARM Cloud Radar." Preprints, 32th International Conference on Radar Meteorology, American Meteorological Society, Albuquerque, NM.

Mudukutore AS, Chandrasekar V, Keeler RJ. 1998. "Pulse Compression for Weather Radar." IEEE Transactions on Geoscience and Remote Sensing 36, 1. 
OHora F, and J Bech. 2005. "Operational Use of Pulse Compression in Weather Radars." Preprints, 32th International Conference on Radar Meteorology, American Meteorological Society, Albuquerque, NM.

PopStefanija, I, J Knorr, P Buczynski, and R Bluth. 2005. “Advance Weather Survaillance Algorithms and Techniques using a Rapid Scanning X-band Radar - First Results." Preprints, 32th International Conference on Radar Meteorology, American Meteorological Society, Albuquerque, NM.

Rutledge SA, R Cifelli, T Lang, S Nesbitt, KS Gage, CR Williams, B Martner, S Matrosov, V Bringi, and PC Kennedy. 2005. "The Front Range Pilot Project for GPM: An instrument and concept test."

Preprints, 32th International Conference on Radar Meteorology, American Meteorological Society, Albuquerque, NM.

Serafin, RJ, and JW Wilson. 2000. "Operational weather radar in the United States: Progress and opportunity." Bulletin of the American Meteorological Society, 81, 501-518.

Weber, ME, J Cho, J Flavin, J Herd, and M Vai. 2005. "Multi-Function Phased-Array Radar for U.S. Civil-Sector Surveillance Needs." Preprints, 32th International Conference on Radar Meteorology, Amer. Meteorological Society, Albuquerque, NM. 